\title{
Self-Esteem, Happiness, and Flourishing in Times of COVID-19: A Study During the Lockdown Period in Ecuador
}

\begin{abstract}
Clara Paz ${ }^{1}$, Carlos Hermosa-Bosano ${ }^{1}$, Paula Hidalgo-Andrade ${ }^{1 *}$, Javier Garcia-Manglano ${ }^{2}$, Charo Sábada Chalezquer ${ }^{3}$, Claudia López-Madrigal ${ }^{4}$ and Cecilia Serrano ${ }^{5}$

${ }^{1}$ School of Psychology and Education, Universidad de Las Américas, Quito, Ecuador, ${ }^{2}$ Institute for Culture and Society, University of Navarra, Pamplona, Spain, ${ }^{3}$ School of Communication, University of Navarra, Pamplona, Spain, ${ }^{4}$ School of Education and Psychology, University of Navarra, Pamplona, Spain, ${ }^{5}$ Department of Sociology, Università Cattolica di Milano, Milano, Italy
\end{abstract}

\section{OPEN ACCESS}

Edited by:

Franco Mascayano, Columbia University Irving Medical

Center, United States

Reviewed by: Edgar Pazmiño, Instituto Psiquiátrico Sagrado Corazión, Ecuador Tintin Sukartini,

Airlangga University, Indonesia

*Correspondence: Paula Hidalgo-Andrade paula.hidalgo@udla.edu.ec

This Original Article is part of the IJPH Special Issue "The Impact of the COVID-19 Pandemic on Mental Health."

Received: 19 August 2021 Accepted: 03 February 2022 Published: 25 February 2022

Citation: Paz C, Hermosa-Bosano C, HidalgoAndrade P, García-Manglano J, Sábada Chalezquer C, López-Madrigal $C$ and Serrano C (2022) Self-Esteem, Happiness, and Flourishing in Times of COVID-19: A Study During the Lockdown Period in Ecuador. Int J Public Health 67:1604418. doi: 10.3389/ijph.2022.1604418
Objective: Several studies have investigated the negative toll the pandemic has had on people's mental health. However, there is limited research on the pandemic's effect on positive mental health variables. This article reports on the levels of self-esteem and wellbeing (flourishing and happiness) in a sample of adults living in Ecuador and their relationships with the characteristics of their personal situation and the effects of the COVID-19 pandemic had on their personal lives.

Methods: A total of 766 adults completed an anonymous online survey between March and August 2020.

Results: Participants reported average scores in the flourishing scale, the majority considered themselves to be happy or very happy people, and more than half presented high levels of self-esteem. Age, education, socioeconomic status, time spent using mobile phones and on hobbies, among others, explained self-esteem, happiness, and flourishing.

Conclusion: The relationships between sociodemographic and situational variables of confinement during the pandemic are discussed, as well as the possible predictors of happiness, flourishing, and self-esteem.

Keywords: COVID-19, Ecuador, flourishing, happiness, self-esteem, well-being

\section{INTRODUCTION}

The coronavirus pandemic (COVID-19) has altered the lifestyle of many people. Since the beginning of the pandemic, many countries -including Ecuador- adopted various strategies to protect their populations and reduce infections and deaths. These measures included mandatory confinement, curfew, mobility restrictions, teleworking, remote education, 
mandatory use of masks, limitation of capacity in restaurants and commercial spaces, restrictions in bars and nightlife centers, among others [1].

Evidence indicates that the longer people are in isolation or confinement, the greater the negative effects on their mental health [2]. Several studies in Ecuador [3, 4] and around the world $[5,6]$ report these detrimental effects on the mental health of people because of the pandemic. Most psychological research has focused on exploring the negative consequences of restrictive measures, including symptoms of stress, psychological distress, confusion, and anger [2].

However, to gain a better understanding of people's wellbeing and mental health during this health crisis, it is imperative to analyze its potential effects on other aspects of the health spectrum, such as the levels of self-esteem, happiness, and flourishing. Self-esteem is positively related to general well-being and negatively related to stress, anxiety, and depression [7]. Studies during the COVID-19 pandemic have found that self-esteem could be an anxietybuffer which can also protect from the impact of other factors, such as depression and loneliness [8].

Another relevant construct in positive psychology is happiness. Happiness allows noticing and taking advantage of positive behaviors and circumstances [9] and relates to better mental and physical health [10]. Studies conducted during the pandemic show correlations between happiness and other well-being variables. For example, a longitudinal study found that a decrease in happiness predicted a decrease in perceived health and was associated with a decreased perceived quality of life [11].

Moreover, it is known that the mere absence of illness is neither a determinant of well-being nor an indicator of good mental health [12]. The presence of mental health is necessary to attain integral well-being, which has been defined as flourishing or prosperity. Despite some differences in its definition, a flourished person is characterized for having high levels of subjective wellbeing [13] and for being functional, both psychologically and socially, through positive relationships, meaning in life, self-acceptance, and self-esteem [14]. During the pandemic, a study showed that there was a negative relationship between COVID-19 fear and human flourishing because of selfisolation and restricted activities, particularly during the lockdown periods [15].

This article seeks to report the levels of self-esteem and wellbeing (flourishing and global happiness) experienced by people living in Ecuador during the months of quarantine due to the COVID-19 pandemic. We consider that self-esteem, global happiness, and flourishing may act as protective factors against the possible negative consequences of the pandemic. Also, understanding how sociodemographic characteristics and personal situations are related to these variables will allow the design and implementation of effective interventions or other kinds of support from a positive psychology perspective.

\section{METHODS}

\section{Design and Participants}

This article analyzes data from 766 individuals who reported being in Ecuador at the time of the study (March to August 2020). This research is part of a non-experimental, cross-sectional, quantitative study carried out in 11 Spanish-speaking countries (Welfare in quarantine: https:/en.unav.edu/web/ culture-and-society-institute/youth-in-transition/research/bienestarcuarentena-coronavirus-tecnologia). To participate, individuals had to be 18 years old or older, indicate the country in which they were living during the lockdown, and complete an anonymous online survey.

\section{Instruments}

The following information was analyzed in this study:

\section{Sociodemographic Data}

This section included self-report questions on sex, age, highest level of education, as well as employment, socioeconomic, marital, and relationship status (i.e., with or without a steady partner).

\section{Personal Living Conditions During COVID-19}

This section inquired about the number of people with whom the respondent was living in the same household and how many of them were older than 60 years or younger than 12 years of age. We also inquired about whether the respondent was in the highest risk group for COVID-19 (due to age or pre- existing illness), whether there were changes in his/her work situation due to the pandemic (loss of a job or not), the number of weekly outings during the quarantine, and the perceived increase in time spent using cell phones, doing hobbies, and conducting physical exercise.

\section{COVID-19 Effects}

This section included questions on whether the respondent, or a person close to them had had symptoms, had received a positive diagnosis, had been hospitalized, or had died from COVID-19.

\section{Self-Esteem Scale}

We used the Rosenberg Scale [16], validated in Spanish [7] in this study. This questionnaire consists of 10 items using a Likert-type scale from $1=$ not at all to $4=$ very much. Some examples of its statements are "I feel that I'm a person of worthy, at least on an equal plane with others" and "On the whole, I am satisfied with myself." After reversing five items and summing the total score, responses are classified into low [10-25], medium [26-29], and high (30-40) levels of self-esteem. In this study, this scale showed a reliability of 0.85 .

\section{Global Happiness}

To explore perceived global happiness, respondents were asked: "Do you consider yourself a happy person?" Response options ranged from $1=$ not at all to $4=$ very much. 


\section{Flourishing}

The Flourishing Scale [17] was used in its Spanish version [18]. This scale assesses social and psychological flourishing through the perception of success in relationships, self-esteem, purpose in life, and optimism. It has eight items with Likert-type response options (1 $=$ not at all to $4=$ very much). The overall score indicates the psychological well-being corresponding to flourishing, and it is obtained from the sum of all responses. The total score ranges from 8 to 32 . The higher the total score, the higher the levels of wellbeing. Cronbach's alpha of this scale was 0.89 .

\section{Procedure}

The study received approval from the ethics committee of the University of Navarra (2020.087) before data collection. The survey was created in Google Forms and the link was distributed through the social networks of the researchers and the research teams involved; an email was also sent through the distribution lists of the university of the research group in Ecuador, after obtaining the respective permissions. The informed consent protocol, specifying the details of the research and the voluntariness and anonymity of participation, was shown on the first page of the survey. Data were collected during the highest peak of the first wave of the COVID-19 pandemic in Ecuador between March and August 2020.

\section{Data Analysis}

Descriptive analyses were performed for the sample and the study variables. Pearson's correlations were run for age, number of people in the same household, number of people over 60 and under 12 years old living in the same household. In addition, according to the type of variable, different analyses were performed to determine the univariate relationships and differences on the study variables (selfesteem, happiness, and flourishing) based on the sociodemographic variables (sex, higher level of education achieved, employment, socioeconomic, marital, and relationship status), the situational context of confinement (whether the respondent was in the highest risk group for COVID-19, whether the participant had lost his/her job, the number of weekly outings, and the perceived increase in time spent using cell phones, hobbies, and physical exercise), and the effects of COVID-19 (whether the respondent or a close person had symptoms, had a positive diagnosis, had been hospitalized, or had died from COVID-19). Given that self-esteem and happiness were codified by levels, Chi-squares were performed to identify potential differences. Due to its continuous nature, ANOVAs were conducted when analyzing the flourishing scores. Finally, for each of the three main variables, linear regressions were performed placing them as dependent variables and including, as independent variables, those that were shown to be significant in the previous analyses. All analyses were carried out using SPSS version 25.

\section{RESULTS}

\section{Sample Characteristics}

Of the total sample $(N=766), 274$ were men and 492 women. Participants' age ranged from 18 to 85 years $(M=32.35, S D=$ 28.0). Table 1 shows their sociodemographic details.
TABLE 1 | Sociodemographic data of the sample $(N=766)$ Self-esteem, happiness and flourishing in times of COVID-19: A study during the lockdown period in Ecuador, Ecuador, 2020.

\begin{tabular}{|c|c|c|}
\hline & $\underline{n}$ & $\%$ \\
\hline \multicolumn{3}{|l|}{ Sex } \\
\hline Men & 274 & 35.8 \\
\hline Women & 492 & 64.2 \\
\hline \multicolumn{3}{|l|}{ Age (by groups) } \\
\hline $18-22$ & 193 & 25.2 \\
\hline 23-29 & 219 & 28.6 \\
\hline 30-39 & 153 & 20.0 \\
\hline $40-49$ & 98 & 12.8 \\
\hline $50-59$ & 76 & 9.9 \\
\hline Over 60 & 27 & 3.5 \\
\hline \multicolumn{3}{|l|}{ Education } \\
\hline High school diploma or less & 203 & 26.5 \\
\hline Professional or technical training & 83 & 10.8 \\
\hline Higher education & 480 & 62.7 \\
\hline \multicolumn{3}{|l|}{ Employment status } \\
\hline Study & 233 & 30.4 \\
\hline Study and work & 126 & 16.4 \\
\hline Work & 257 & 33.6 \\
\hline Unemployed & 88 & 11.5 \\
\hline Retired & 13 & 1.7 \\
\hline Other & 49 & 6.4 \\
\hline \multicolumn{3}{|l|}{ Socioeconomic status } \\
\hline Low & 92 & 12.0 \\
\hline Medium & 435 & 56.8 \\
\hline High & 239 & 31.2 \\
\hline \multicolumn{3}{|l|}{ Marital status } \\
\hline Single with no children & 447 & 58.4 \\
\hline Single with children & 36 & 4.7 \\
\hline Separated or divorced & 50 & 6.5 \\
\hline Widower & 4 & 0.5 \\
\hline Married or in a civil union & 229 & 29.9 \\
\hline \multicolumn{3}{|l|}{ Couple situation } \\
\hline Without a stable partner & 537 & 70.1 \\
\hline With a stable partner & 229 & 29.9 \\
\hline
\end{tabular}

Most participants ( $n=649,84.7 \%)$ reported spending the lockdown period with others in the same household. The number of people they were living with ranged from 1 to $14(M d n=3$, $I Q R=2$ ). Of the total number of respondents who indicated living with people over 60 years $(n=297,38.8 \%)$, the majority reported living with one or two people. On the other hand, of the participants who lived with children under 12 years $(n=235$, $30.7 \%), 160$ (20.9\%) lived with one child, 52 (6.8\%) with two children, and $23(3.0 \%)$ with three children. Table 2 shows the details of participants' personal living conditions.

In addition, as seen in Table 3, most participants reported having family members or acquaintances who had experienced symptoms of the disease, or who had received a positive diagnosis. However, most participants reported not knowing whether people in their social network had been hospitalized or had died from COVID-19.

\section{Key Variables: Self-Esteem, Happiness, and Flourishing}

We analyzed the potential relationships between the variables of interest and the sociodemographic, situational, and 
TABLE 2 | Personal situations during the quarantine by COVID-19. Self-esteem, happiness and flourishing in times of COVID-19: A study during the lockdown period in Ecuador, Ecuador, 2020.

\begin{tabular}{|c|c|c|}
\hline & $n$ & $\%$ \\
\hline \multicolumn{3}{|c|}{ Impact of COVID-19 on job stability } \\
\hline Lost job & 41 & 5.4 \\
\hline Did not lose the job & 725 & 94.6 \\
\hline \multicolumn{3}{|c|}{ Weekly times leaving the house during quarantine } \\
\hline None & 308 & 40.2 \\
\hline Once or twice & 374 & 48.8 \\
\hline Three or more times & 84 & 11.0 \\
\hline \multicolumn{3}{|c|}{ Increased use of cellular phones } \\
\hline Nothing & 18 & 2.3 \\
\hline Little & 186 & 24.3 \\
\hline Very & 422 & 55.1 \\
\hline Much & 140 & 18.3 \\
\hline \multicolumn{3}{|c|}{ Changes in the time dedicated to physical exercise } \\
\hline It has decreased & 349 & 45.6 \\
\hline It has remained the same & 209 & 27.3 \\
\hline It has increased & 208 & 27.2 \\
\hline \multicolumn{3}{|c|}{ Changes in time spent on hobbies } \\
\hline It has decreased & 281 & 36.7 \\
\hline It has remained the same & 205 & 26.8 \\
\hline It has increased & 280 & 36.6 \\
\hline \multicolumn{3}{|c|}{ Person at risk due to age or previous pathology } \\
\hline Yes & 119 & 15.5 \\
\hline No & 647 & 84.5 \\
\hline \multicolumn{3}{|l|}{ Cohabitation with other people } \\
\hline Yes & 649 & 84.7 \\
\hline No & 117 & 15.3 \\
\hline \multicolumn{3}{|c|}{ Cohabitation with people over 60 years of age } \\
\hline Yes & 297 & 38.8 \\
\hline No & 469 & 61.2 \\
\hline \multicolumn{3}{|c|}{ Cohabitation with children under 12 years of age } \\
\hline Yes & 235 & 30.7 \\
\hline No & 531 & 69.3 \\
\hline
\end{tabular}

pandemic-related variables. Table 4 indicates the levels of selfesteem, global happiness, and flourishing according to the sociodemographic variables and Table $\mathbf{5}$ shows these key variables according to the context variables.

\section{Self-Esteem}

On average, the sample presented high levels of self-esteem $(M=$ $30.3, S D=5.4)$. When analyzing self-esteem by categorial levels, results shown that slightly more than half of the sample $(n=454$, $59.3 \%)$ presented high levels of self-esteem, whereas the rest presented medium $(n=171,22.3 \%)$ and low $(n=141,18.4 \%)$ levels. When analyzing its relationship to the sociodemographic variables, sex was not significant, but age was positively correlated $(r=0.22, p<0.001)$. It was observed that people between 18 and 22 years of age had lower self-esteem than the rest of the age groups, $\chi^{2}(10, N=766)=54.67, p<0.001$. A significant relationship was also observed with the education level. People with high school education or less $(n=203)$ had lower levels of self-esteem (29.06\% low, $27.59 \%$ medium and $43.35 \%$ high) than those with professional or technical training ( $n=83 ; 13.25 \%$ low, $19.28 \%$ medium and $67.47 \%$ high) and those with higher education ( $n=480 ; 14.79 \%$ low, $20.63 \%$ medium and $64.58 \%$ high $), \chi^{2}=(4, N=766)=32.52, p<0.001$.

In the case of employment status, people who reported only studying had significantly lower levels of self-esteem compared to those who worked and studied, and those who reported only working, $\chi^{2}(10, N=766)=38.68, p<0.001$. In contrast, people with a low socioeconomic status presented significantly lower levels of self-esteem compared to people with high and medium levels, $\chi^{2}(4, N=766)=20.39, p<0.001$.

Regarding marital and relationship status, it was observed that single people without children, $\chi^{2}(8, N=766)=29.67, p<0.001$, and those without a stable partner, $\chi^{2}(2, N=766)=16.77, p<$ 0.001 , had significantly lower levels of self-esteem compared to married people or those with a stable partner.

When analyzing participants' context during COVID-19, only the perceived changes in cell phone use and changes in the amount of time spent on hobbies showed significant relationships with self-esteem. People who claimed to have increased their cell phone usage time by "a lot" presented lower levels of self-esteem compared to those who increased their usage by "a little" and "quite a lot" $\chi^{2}(6, N=766)=12.99$, $p=0.04$. People who reported having decreased their time dedicated to hobbies also showed significantly lower levels of self-esteem compared to those who reported an increase on it, $\chi^{2}$ $(4, N=766)=10.54, p=0.03$. On the other hand, knowing people directly affected by COVID-19 or presenting these effects oneself (having symptoms, confirmed diagnosis, hospitalization) did not have statistically significant relationships.

Finally, we conducted a linear regression using variables that were significantly associated with self-esteem as independent factors. After performing the first regression model, the variables marital status $(\beta=-0.014, p=0.89)$, sentimental situation $(\beta=0.043, p=0.97)$ and cell phone use $(\beta=-0.057$, $p=0.11)$ were excluded. The model that significantly explained self-esteem $\left[F(5,760)=14.54, p<0.001, R_{\text {adjusted }=0.081] \text { included }}^{2}\right.$ age $(\beta=0.164, p<0.001)$, education $(\beta=0.103, p=0.007)$, employment status $(\beta=-0.084, p=0.02)$, socioeconomic status $(\beta=0.098, p=0.005)$, and time spent in hobbies $(\beta=0.095, p=$ $0.007)$.

TABLE 3 | People affected by COVID-19. Self-esteem, happiness and flourishing in times of COVID-19: A study during the lockdown period in Ecuador, Ecuador, 2020.

\begin{tabular}{|c|c|c|c|c|}
\hline & With symptoms & With diagnosis & Hospitalized & Deceased \\
\hline & $n(\%)$ & $n(\%)$ & $n(\%)$ & $n(\%)$ \\
\hline Respondent & 99 (12.9) & $64(8.4)$ & $4(0.5)$ & - \\
\hline Family & $535(69.8)$ & $511(66.7)$ & $49(6.4)$ & $32(4.2)$ \\
\hline Acquaintances & $98(12.8)$ & $144(18.8)$ & $181(23.6)$ & $146(19.1)$ \\
\hline Nobody & $34(4.4)$ & $47(6.1)$ & $532(69.5)$ & $588(76.8)$ \\
\hline
\end{tabular}


TABLE 4 | Self-esteem, happiness, and flourishing according to sociodemographic variables. Self-esteem, happiness and flourishing in times of COVID-19: A study during the lockdown period in Ecuador, Ecuador, 2020.

\begin{tabular}{|c|c|c|c|c|c|c|c|c|c|}
\hline & \multirow[t]{3}{*}{$n$} & \multicolumn{3}{|c|}{ Self-esteem } & \multicolumn{4}{|c|}{ Consideration as a happy person } & \multirow{3}{*}{$\frac{\text { Flourishing }}{M(S D)}$} \\
\hline & & \multirow{2}{*}{$\frac{\text { Low }}{\%}$} & \multirow{2}{*}{$\frac{\text { Medium }}{\%}$} & \multirow{2}{*}{$\frac{\text { High }}{\%}$} & \multirow{2}{*}{$\frac{\text { Nothing }}{\%}$} & \multirow{2}{*}{$\frac{\text { Little }}{\%}$} & \multirow{2}{*}{$\frac{\text { Very }}{\%}$} & \multirow{2}{*}{$\frac{\text { Much }}{\%}$} & \\
\hline & & & & & & & & & \\
\hline Total & 766 & 18.4 & 22.3 & 59.3 & 1.3 & 16.8 & 59.1 & 22.7 & $6.89(1.82)$ \\
\hline \multicolumn{10}{|l|}{ Sociodemographics } \\
\hline \multicolumn{10}{|l|}{ Sex } \\
\hline Male & 274 & 6.79 & 22.26 & 60.95 & 2.19 & 15.33 & 58.03 & 24.45 & $6.86(1.81)$ \\
\hline Female & 492 & 19.31 & 22.36 & 58.33 & 0.81 & 17.68 & 59.76 & 21.75 & $6.91(1.83)$ \\
\hline \multicolumn{10}{|l|}{ Age (by groups) } \\
\hline $18-22$ & 193 & 29.53 & 26.94 & 43.52 & 2.07 & 20.73 & 61.14 & 16.06 & $6.63(1.82)$ \\
\hline 23-29 & 219 & 20.55 & 18.26 & 61.19 & 0.91 & 18.72 & 57.53 & 22.83 & $7.03(1.88)$ \\
\hline $30-39$ & 153 & 14.38 & 29.41 & 56.21 & 1.31 & 18.95 & 59.48 & 20.26 & $6.74(1.82)$ \\
\hline $40-49$ & 98 & 10.20 & 12.24 & 77.55 & 2.04 & 11.22 & 53.06 & 33.67 & $7.09(1.75)$ \\
\hline $50-59$ & 76 & 7.89 & 22.37 & 69.74 & 0.00 & 6.58 & 65.79 & 27.63 & $703(1.65)$ \\
\hline Over 60 & 27 & 3.70 & 18.52 & 77.78 & 0.00 & 11.11 & 59.26 & 29.63 & $7.44(1.83)$ \\
\hline \multicolumn{10}{|l|}{ Education } \\
\hline High school diploma or less & 203 & 29.06 & 27.59 & 43.35 & 2.96 & 19.70 & 59.11 & 18.23 & $6.59(1.84)$ \\
\hline Professional or technical training & 83 & 13.25 & 19.28 & 67.47 & 0.00 & 13.25 & 65.06 & 21.69 & $6.73(1.65)$ \\
\hline Higher education & 480 & 14.79 & 20.63 & 64.58 & 0.83 & 16.25 & 58.13 & 24.79 & $7.05(1.82)$ \\
\hline \multicolumn{10}{|l|}{ Employment status } \\
\hline Study & 233 & 27.90 & 24.46 & 47.64 & 1.72 & 21.46 & 62.66 & 14.16 & $6.71(1.8)$ \\
\hline Study and work & 126 & 15.08 & 15.87 & 69.05 & 1.59 & 13.49 & 60.32 & 24.60 & $7.19(1.79)$ \\
\hline Work & 257 & 11.28 & 21.01 & 67.70 & 0.39 & 13.62 & 57.20 & 28.79 & $7.14(1.72)$ \\
\hline Unemployed & 88 & 19.32 & 27.27 & 53.41 & 3.41 & 19.32 & 59.09 & 18.18 & $6.56(1.99)$ \\
\hline Retired & 13 & 0.00 & 23.08 & 76.92 & 0.00 & 0.00 & 38.46 & 61.54 & $7.98(1.39)$ \\
\hline Other & 49 & 22.45 & 26.53 & 51.02 & 0.00 & 20.41 & 55.10 & 24.49 & $6.0(1.74)$ \\
\hline \multicolumn{10}{|l|}{ Socioeconomic status } \\
\hline Low & 92 & 33.70 & 26.09 & 40.22 & 1.09 & 31.52 & 57.61 & 9.78 & $6.08(1.78)$ \\
\hline Medium & 435 & 16.55 & 21.84 & 61.61 & 1.38 & 16.09 & 58.85 & 23.68 & $6.9(1.8)$ \\
\hline High & 239 & 15.90 & 21.76 & 62.34 & 1.26 & 12.55 & 60.25 & 25.94 & $7.18(1.78)$ \\
\hline \multicolumn{10}{|l|}{ Marital status } \\
\hline Single with no children & 447 & 24.16 & 22.37 & 53.47 & 1.79 & 19.02 & 59.06 & 20.13 & $6.82(1.85)$ \\
\hline Single with children & 36 & 13.89 & 30.56 & 55.56 & 2.78 & 22.22 & 55.56 & 19.44 & $6.6(1.93)$ \\
\hline Separated or divorced & 50 & 10.00 & 20.00 & 70.00 & 0.00 & 20.00 & 52.00 & 28.00 & $6.6(1.61)$ \\
\hline Widower & 4 & 0.00 & 0.00 & 100.00 & 0.00 & 0.00 & 75.00 & 25.00 & $7.5(1.48)$ \\
\hline Married or with a stable partner & 229 & 10.04 & 21.83 & 68.12 & 0.44 & 11.35 & 61.14 & 27.07 & $7.13(1.77)$ \\
\hline \multicolumn{10}{|l|}{ Couple situation } \\
\hline Without a stable partner & 537 & 21.97 & 22.53 & 55.49 & 1.68 & 19.18 & 58.29 & 20.86 & $6.79(1.83)$ \\
\hline Married or with a stable partner & 229 & 10.04 & 21.83 & 68.12 & 0.44 & 11.35 & 61.14 & 27.07 & $7.13(1.77)$ \\
\hline
\end{tabular}

\section{Global Happiness}

Most respondents considered themselves to be happy people. Responses on this variable ranged from not at all $(n=10,1.3 \%)$, a little $(n=129,16.8 \%)$, quite a lot $(n=453,59.1 \%)$, and very much $(n=174,22.7 \%)$. The sociodemographic variables that showed significant relationships with global happiness levels were age $(r=0.15, p<0.001)$, employment status $\left(\chi^{2}(15, N=766)=37.21\right.$, $p=0.001)$, socioeconomic status $\left(\chi^{2}(6, \mathrm{~N}=766)=22.72\right.$, $p=0.001)$ and sentimental status $\left(\chi^{2}(3, N=766)=10.68\right.$, $p=0.01)$. People who worked and those who were retired reported being happier than those who were studying. Likewise, retired people reported being significantly happier than unemployed people. On the other hand, people with high and medium socioeconomic status reported significantly higher levels of happiness than people with lower socioeconomic status. Finally, married people and those with a stable partner considered themselves happier than people without a stable partner.

Regarding the personal living conditions' variables, only the time dedicated to hobbies was significantly related, $\chi^{2}(6$,
$N=766)=18.53, p=0.005$. People who had decreased their time engaged in hobbies during lockdown presented lower overall happiness compared to those who had increased it.

When performing a linear regression to predict happiness levels, all variables significantly related were included as predictors. Employment $(\beta=-0.050, p=0.16)$ and sentimental status $(\beta=0.043, p=0.30)$ were excluded. The model that significantly explained happiness $[F(3,762)=5.76$, $\left.p<0.001, R_{\text {adjusted }}^{2} 0.047\right]$ included age $(\beta=0.140, p<0.001)$, socioeconomic status $(\beta=0.122, p=0.001)$, and time spent in hobbies $(\beta=0.111, p=0.002)$.

\section{Flourishing}

The sample obtained average levels in the flourishing scale $(M=$ $6.89, S D=1.82)$. Flourishing and age had a positive correlation $(r=0.07, p=0.04)$. Also, people with higher education showed significantly higher levels of flourishing than those with high school education or less $[F(2,763)=5.07, p=0.006]$. Similarly, people who described their work situation as "other" reported 
TABLE 5 | Self-esteem, happiness, and flourishing according to personal situation during quarantine and effects of COVID-19. Self-esteem, happiness and flourishing in times of COVID-19: A study during the lockdown period in Ecuador, Ecuador, 2020.

\begin{tabular}{|c|c|c|c|c|c|c|c|c|c|}
\hline & \multirow[t]{3}{*}{$n$} & \multicolumn{3}{|c|}{ Self-esteem } & \multicolumn{4}{|c|}{ Consideration as a happy person } & \multirow{3}{*}{$\frac{\text { Flourishing }}{M(S D)}$} \\
\hline & & \multirow{2}{*}{$\frac{\text { Low }}{\%}$} & \multirow{2}{*}{$\frac{\text { Medium }}{\%}$} & \multirow{2}{*}{$\frac{\text { High }}{\%}$} & \multirow{2}{*}{$\frac{\text { Nothing }}{\%}$} & \multirow{2}{*}{$\frac{\text { Little }}{\%}$} & \multirow{2}{*}{$\frac{\text { Very }}{\%}$} & \multirow{2}{*}{$\frac{\text { Much }}{\%}$} & \\
\hline & & & & & & & & & \\
\hline Total & 766 & 18.4 & 22.3 & 59.3 & 1.3 & 16.8 & 59.1 & 22.7 & $6.89(1.82)$ \\
\hline \multirow{2}{*}{\multicolumn{10}{|c|}{$\begin{array}{l}\text { Personal situation during quarantine by COVID-19 } \\
\text { Impact of COVID-19 }\end{array}$}} \\
\hline & & & & & & & & & \\
\hline Lost job & 725 & 18.34 & 22.07 & 59.59 & 1.10 & 16.55 & 59.59 & 22.76 & $6.92(1.8)$ \\
\hline Did not lose the job & 41 & 19.51 & 26.83 & 53.66 & 4.88 & 21.95 & 51.22 & 21.95 & $6.37(2.03)$ \\
\hline \multicolumn{10}{|c|}{ Weekly times leaving the house during quarantine } \\
\hline None & 308 & 21.10 & 20.78 & 58.12 & 0.97 & 18.51 & 57.47 & 23.05 & $7.06(1.9)$ \\
\hline Once or twice & 374 & 18.45 & 22.46 & 59.09 & 1.34 & 16.31 & 59.09 & 23.26 & $6.80(1.81)$ \\
\hline Three or more times & 84 & 8.33 & 27.38 & 64.29 & 2.38 & 13.10 & 65.48 & 19.05 & $6.69(1.5)$ \\
\hline \multicolumn{10}{|c|}{ Increased use of cellular phones } \\
\hline Nothing & 18 & 22.22 & 27.78 & 50.00 & 0.00 & 27.78 & 55.56 & 16.67 & $7.11(1.83)$ \\
\hline Little & 186 & 13.98 & 20.97 & 65.05 & 0.54 & 15.59 & 60.22 & 23.66 & $6.97(1.8)$ \\
\hline Very & 422 & 17.54 & 21.56 & 60.90 & 1.42 & 15.17 & 62.56 & 20.85 & $6.84(1.47)$ \\
\hline Much & 140 & 26.43 & 25.71 & 47.86 & 2.14 & 22.14 & 47.86 & 27.86 & $6.93(2.07)$ \\
\hline \multicolumn{10}{|c|}{ Changes in time spent exercising } \\
\hline It has decreased & 349 & 19.20 & 22.92 & 57.88 & 2.01 & 20.63 & 55.87 & 21.49 & $6.74(1.92)$ \\
\hline It has remained the same & 209 & 18.18 & 20.57 & 61.24 & 0.96 & 14.35 & 60.29 & 24.40 & $7.08(1.71)$ \\
\hline It has increased & 208 & 17.31 & 23.08 & 59.62 & 0.48 & 12.98 & 63.46 & 23.08 & $6.96(1.74)$ \\
\hline \multicolumn{10}{|c|}{ Changes in time spent on hobbies } \\
\hline It has decreased & 281 & 23.13 & 22.42 & 54.45 & 1.07 & 23.49 & 56.94 & 18.51 & $6.65(1.86)$ \\
\hline It has remained the same & 205 & 16.59 & 25.85 & 57.56 & 1.95 & 12.68 & 63.41 & 21.95 & $6.85(1.79)$ \\
\hline It has increased & 280 & 15.00 & 19.64 & 65.36 & 1.07 & 13.21 & 58.21 & 27.50 & $7.17(1.77)$ \\
\hline Person at risk due to age or & ous pa & & & & & & & & \\
\hline Yes & 119 & 11.76 & 20.17 & 68.07 & 0.84 & 17.65 & 53.78 & 27.73 & $6.95(1.89)$ \\
\hline No & 647 & 19.63 & 22.72 & 57.65 & 1.39 & 16.69 & 60.12 & 21.79 & $6.88(1.81)$ \\
\hline Cohabitation with other peor & & & & & & & & & \\
\hline Yes & 649 & 19.26 & 22.50 & 58.24 & 1.39 & 17.57 & 57.32 & 23.73 & $6.88(1.83)$ \\
\hline No & 117 & 13.68 & 21.37 & 64.96 & 0.85 & 12.82 & 69.23 & 17.09 & $6.93(1.79)$ \\
\hline Cohabitation with people ov & years o & & & & & & & & \\
\hline Yes & 297 & 20.20 & 24.24 & 55.56 & 1.68 & 19.87 & 56.57 & 21.89 & 6.79 (1.83) \\
\hline No & 469 & 17.27 & 21.11 & 61.62 & 1.07 & 14.93 & 60.77 & 23.24 & $6.96(1.81)$ \\
\hline Cohabitation with children ur & 2 years & & & & & & & & \\
\hline Yes & 235 & 19.15 & 22.98 & 57.87 & 2.55 & 17.87 & 54.47 & 25.11 & $6.82(1.99)$ \\
\hline No & 531 & 18.08 & 22.03 & 59.89 & 0.75 & 16.38 & 61.21 & 21.66 & $6.93(1.74)$ \\
\hline People, close to respondent & ted by & D-19 & & & & & & & \\
\hline With symptoms & & & & & & & & & \\
\hline Respondent & 99 & 20.20 & 24.24 & 55.56 & 1.01 & 18.18 & 58.59 & 22.22 & $6.98(1.87)$ \\
\hline Family & 535 & 18.69 & 19.81 & 61.50 & 1.68 & 15.33 & 60.37 & 22.62 & 7.05 (1.83) \\
\hline Known & 98 & 13.27 & 28.57 & 58.16 & 0.00 & 19.39 & 55.10 & 25.51 & $6.49(1.61)$ \\
\hline Nobody & 34 & 23.53 & 38.24 & 38.24 & 0.00 & 29.41 & 52.94 & 17.65 & 5.64 (1.39) \\
\hline With diagnosis & & & & & & & & & \\
\hline Respondent & 64 & 20.31 & 28.13 & 51.56 & 0.00 & 17.19 & 62.50 & 20.31 & $6.83(1.75)$ \\
\hline Family & 511 & 18.98 & 20.16 & 60.86 & 1.96 & 15.66 & 60.86 & 21.53 & 7.05 (1.81) \\
\hline Known & 144 & 13.19 & 24.31 & 62.50 & 0.00 & 17.36 & 54.17 & 28.47 & $6.74(1.82)$ \\
\hline Nobody & 47 & 25.53 & 31.91 & 42.55 & 0.00 & 27.66 & 51.06 & 21.28 & $5.68(1.50)$ \\
\hline Hospitalized & & & & & & & & & \\
\hline Respondent & 4 & 25.00 & 75.00 & 0.00 & 0.00 & 50.00 & 50.00 & 0.00 & $5.0(1.36)$ \\
\hline Family & 49 & 16.33 & 18.37 & 65.31 & 0.00 & 18.37 & 55.10 & 26.53 & 7.22 (1.89) \\
\hline Known & 181 & 14.36 & 22.10 & 63.54 & 0.55 & 13.26 & 56.91 & 29.28 & 7.01 (1.89) \\
\hline Nobody & 532 & 19.92 & 22.37 & 57.71 & 1.69 & 17.67 & 60.34 & 20.30 & $6.84(1.78)$ \\
\hline Deceased & & & & & & & & & \\
\hline Family & 32 & 18.75 & 21.88 & 59.38 & 0.00 & 21.88 & 56.25 & 21.88 & 7.21 (1.96) \\
\hline Known & 146 & 13.70 & 22.60 & 63.70 & 0.68 & 12.33 & 56.16 & 30.82 & 6.99 (1.92) \\
\hline Nobody & 588 & 19.56 & 22.28 & 58.16 & 1.53 & 17.69 & 60.03 & 20.75 & 6.85 (1.79) \\
\hline
\end{tabular}

significantly lower levels of flourishing compared to those who are both studying and working and only working $[F(5,760)=$ 6.07, $p<0.001]$. People with a low socioeconomic status presented lower levels of flourishing than those in high and medium levels $[F(2,763)=12.58, p<0.001]$. Finally, people without a stable partner indicated lower levels of flourishing than those who were married or had a stable partner $[F(1,764)=5.81$, $p=0.02]$ 
Concerning the personal living conditions during lockdown, although the student's $t$-test was not significant between living alone or not, the number of people living in the same household showed a significant negative correlation with flourishing $(r=-0.08, p=0.02)$. There were lower levels of flourishing when participants reported living with more people. On the other hand, people who had decreased the time spent on their hobbies presented lower levels of flourishing than those who had increased it $[F(2,763)=5.91, p=0.003]$.

When analyzing the levels of flourishing according to the COVID-19 effects' variables, the results indicated that people who did not know anyone with symptoms presented lower levels of flourishing than those who had a family member with symptoms or those who themselves presented symptoms. Similarly, people who had a family member with symptoms had higher levels of flourishing than those who knew someone (a non-family member) in that situation $[F(3,762)=8.47, p<$ 0.001]. Regarding COVID-19 diagnosis, people who did not know anyone with a confirmed diagnosis had significantly lower levels of flourishing compared to those who had a family member, an acquaintance, or those who had the diagnosis themselves $[F(3,762)=8.89, p<0.001]$. Knowing someone who had required hospitalization or who had died was not significant related to the levels of flourishing.

Finally, when running the linear regression, non-significant variables were excluded, age $(\beta=0.021, p=0.62)$, education $(\beta=$ $0.059, p=0.13)$, sentimental situation $(\beta=0.052, p=0.20)$, number of people living in the same household $(\beta=-0.051, p=0.15)$, and COVID-19 symptoms $(\beta=-0.034, p=0.56)$. The model that significantly explained flourishing $[F(4,761)=16.33, p<0.001$, $\left.R_{\text {adjusted }}^{2}=0.074\right]$ included employment status $(\beta=-0.140, p<0.001)$, socioeconomic status $(\beta=0.147, p<0.001)$, time spent in hobbies ( $\beta$ $=0.113, p=0.001)$, and knowing a person with a diagnosis of COVID-19 $(\beta=-146, p<0.001)$.

\section{DISCUSSION}

This study analyzed the self-esteem and the well-being (flourishing and happiness) of people living in Ecuador during lockdown due to the COVID-19 pandemic. Like studies conducted before the pandemic in the Ecuadorian population, our sample presented high levels of self-esteem. For example, one conducted in 2017 [19] found similar overall levels of self-esteem $(M=31.7)$ to those found in our study $(M=30.3)$, and highlighted self-esteem as a protective factor against meeting the criteria for a major depressive episode, panic disorder, and suicide risk [19].

Regarding happiness, unlike studies that report a decrease in happiness and optimism [20] and similar to others in the same cultural context [21], most respondents in our study considered themselves to be happy people. This result is not surprising given that the 2021 World Happiness Report shows an increase in sadness and worry globally, but an stable overall life evaluations and happiness ranking [22]. Also, according to the World Happiness Index, in 2020 Ecuador ranked in 58th place of the 149 countries [23].
Unlike a cross-sectional study in the United States that found that COVID-19 fear significantly decreased human flourishing and that gender moderated the relationship between those two variables [15], people from our sample showed average levels of flourishing. Longitudinal research may also support our findings. For example, a study that evaluated differences in mental health before and during the pandemic showed that, although there was a decline in mental health indicators -including flourishingduring the initial stages of the lockdown measures, they were not significant when compared to the changes from previous years [24]. Maybe, during the pandemic, people had to adapt and learn to manage their circumstances and achieved environmental mastery and personal growth, characteristics related to flourishing [25]. Research also suggests that people who experience this growth, highlight the opportunities that may arise during life transitions [25]. Results from the current study could show similar pathways.

Regarding sociodemographic and context variables, research shows that there is no definitive evidence linking them as risk factors for mental health problems during quarantine [2], although some have been found that being younger, having lower levels of education, being female, and having a child [26] are features related to greater psychological distress.

The results of our study can be contrasted with others conducted in previous quarantines and with current others about psychological distress that indicate that younger people have higher levels of stress, anxiety, and depression [27]. In concordance with this body of research, we found that the older the age, the higher the self-esteem, the greater the overall happiness, and the higher the levels of flourishing. On the other hand, lower levels of education were related to lower self-esteem and lower levels of flourishing. Likewise, people with low socioeconomic status had significantly lower levels of selfesteem, happiness, and flourishing. Such results highlight the importance of the socio-cultural determinants of health and the importance of shifting our perspective to see the COVID-19 situation as a syndemic rather than a pandemic [28]. This way, we could consider the interaction between social and biological factors to better face this disease and its consequences.

Single people without children and those without a stable partner presented lower self-esteem. The latter also considered themselves to be less happy and present lower levels of flourishing. Concerning occupational status, people who were only studying presented lower self-esteem and overall happiness than those who had a job. This finding complements another study [29] where students presented greater psychological distress. A possible explanation for this result may be that the change of study modality (from face-to-face to virtual) confronted students with a lifestyle in which not only their social contact was modified but also their access to education, with institutions and teachers not fully prepared for this abrupt change.

Although social contact is important, the results indicated that there are lower levels of flourishing when more people live in the same physical space. This may be due to the long duration of confinement and restrictions in the country. In addition, it can be attributed to the fact that being in the same household with many 
people means sharing spaces and resources without the possibility of external distractions or time away from home, which can result in the emergence of interpersonal conflicts. Thus, being in the same physical space might not translate into having social support or positive social interactions.

During this pandemic, there has been an insistence on voluntary confinement, mandatory lockdowns, and physical distancing to curb contagion. This shift towards carrying out daily activities remotely encouraged greater use of technology. However, we found that people who increased the time they spent using cell phones had lower levels of self-esteem than those who perceived that they were using them the same amount of time as before the pandemic. Although the Internet can be a convenient way to stay in touch with others, excessive use may have more negative than positive effects. This result is in line with several previous studies that relate screen time to lower personal wellbeing and depressive [30] and anxious symptomatology [31].

On the contrary, people who reported an increase in the amount of time spent on hobbies presented higher self-esteem and better levels of flourishing. These results are consistent with previous studies that relate leisure activities with better mental health [32] and lower symptoms of depression [33] and anxiety [34] in the adult population. They are also related to recommendations made at the beginning of the pandemic to mitigate the possible negative effects of social distancing and quarantine [35].

In addition to the confinement and other restriction measures, many people have directly experienced the effects of COVID-19. Despite knowing someone with COVID-19 symptoms was a predictor of lower flourishing, those who indicated that they did not know anyone with symptoms or with a confirmed diagnosis had lower levels of flourishing than those who had a family member with symptoms. Also, knowing someone who had been hospitalized or someone who had died from COVID-19 were not related to flourishing levels. People who have not experienced effects on physical health firsthand, or through people close to them, might be more uncertain about the COVID-19 situation and might perceive the measures implemented as more restrictive and meaningless. Likewise, those who know people close to them with symptoms and confirmed diagnosis may feel optimistic about their improvement. A possible explanation for these results derives from the Terror Management Theory [36] which explains the conscious (proximal defenses) and unconscious (distal defenses) mechanisms that people use to protect themselves from death anxiety. Following this theory $[8,37]$, the higher levels of thriving of people who have family members with COVID-19 symptomatology could be distal defenses that decrease unconscious worry about their mortality through life purpose, better relationships, optimism, and improved self-esteem.

This study also provides predictive models for self-esteem, happiness, and flourishing. It was found that age, education, employment status, socioeconomic level, and time spent on hobbies predicted self-esteem. In the case of global happiness, only age, socioeconomic level and time spent on hobbies were significant predictors. Additionally, regarding flourishing, the significant variables were employment status, socioeconomic level, time dedicated to hobbies, and knowing a person diagnosed with
COVID-19. The development of interventions aimed at maintaining and increasing the well-being of the population should consider these characteristics during their design and implementation.

\section{Conclusion and Limitations}

Mental health is more than the absence of disease and the positive aspects of life might be more important than the absence of negative aspects [12]. Thus, this study focused on the positive aspects of mental health during the COVID-19 pandemic. Results showed that, in general, participants had high levels of self-esteem, considered themselves to be fairly or very happy individuals, and had an average level of flourishing. Age, education, socioeconomic status, time spent on mobile phones, and hobbies were found to be relevant to happiness, flourishing, and self-esteem.

Despite the relevance of this study, it is important to recognize its limitations. Although participation was high, having collected data through electronic means may limit the generalizability of the results. Future studies can expand the sample to obtain greater representativeness and include different methods of data collection. Likewise, caution should be exercised when interpreting the data due to their self-report nature. In addition, due to the cross-sectional nature of the study, we cannot establish causal relationships or know the possible longitudinal changes that may have occurred among participants. However, despite its limitations, we are confident that this study contributes to the understanding of aspects of mental health and well-being of the population during an unprecedented global emergency.

Future studies should take these results into account to expand these findings and propose prevention and intervention measures that contribute to promoting mental health by increasing selfesteem, happiness, and flourishing. Particular attention should be paid to the young population with lower levels of well-being. In addition, more should be known about leisure time and dedication to hobbies, which was the only variable that predicted the three constructs of interest. States, governments, and companies could benefit from our findings when analyzing and determining measures for social and economic reactivation.

\section{ETHICS STATEMENT}

The studies involving human participants were reviewed and approved by Ethics committee of University of Navarra (code: 2020.087). The patients/participants provided their written informed consent to participate in this study.

\section{AUTHOR CONTRIBUTIONS}

JG-M, CC, CL-M, and CS contributed to the concept and design of the study, as well as data curation. $\mathrm{CP}, \mathrm{CH}-\mathrm{B}$, and $\mathrm{PH}-\mathrm{A}$ contributed to data collection, interpretation of the data, analysis of results, and manuscript preparation. All authors accept and agree that the work is original, any methods and data presented are described accurately and honestly, and all relevant interests have been disclosed. All authors have read and agreed to the published version of the manuscript. 


\section{FUNDING}

The overall project is promoted by the research group Youth in Transition of the Institute for Culture and Society (ICS) of the University of Navarra and has no external funding.

\section{REFERENCES}

1. Servicio Nacional de Gestión de RiesgosEmergenciasy. Resoluciones COE [Internet]. (2021) Avaliable at: https://www.gestionderiesgos.gob.ec/ resoluciones-coe/.

2. Brooks SK, Webster RK, Smith LE, Woodland L, Wessely S, Greenberg N, et al. The Psychological Impact of Quarantine and How to Reduce it: Rapid Review of the Evidence. The Lancet (2020) 395(10227):912-20. doi:10.1016/s01406736(20)30460-8

3. Paz C, Mascialino G, Adana-Díaz L, Rodríguez-Lorenzana A, Simbaña-Rivera K, Gómez-Barreno L, et al. Anxiety and Depression in Patients with Confirmed and Suspected COVID-19 in Ecuador. Psychiatry Clin Neurosci (2020) 74:554-5. doi:10.1111/pcn.13106

4. Mautong H, Gallardo-Rumbea JA, Alvarado-Villa GE, FernándezCadena JC, Andrade-Molina D, Orellana-Román CE, et al. Assessment of Depression, Anxiety and Stress Levels in the Ecuadorian General Population during Social Isolation Due to the COVID-19 Outbreak: A Cross-Sectional Study. BMC Psychiatry (2021) 21(1):212-5. doi:10.1186/ s12888-021-03214-1

5. Mucci F, Mucci N, Diolaiuti F. Lockdown and Isolation: Psychological Aspects of COVID-19 Pandemic in the General Population. Clin Neuropsychiatry (2020) 17(2):63-4. doi:10.36131/CN20200205

6. Samaniego A, Urzúa A, Buenahora M, Vera-Villarroel P. Sintomatología asociada a trastornos de Salud Mental en trabajadores sanitarios en Paraguay: efecto COVID-19. RIP/IJP (2020) 54(1):e1298. doi:10.30849/ ripijp.v54i1.1298

7. Vázquez Morejón A, García-Bóveda R, Vázquez-Morejón Jiménez R. Escala de autoestima de Rosenberg: fiabilidad y validez en población clínica española. Apunt Psicol (2004) 22(2):247-55. Avaliable at: https://www. apuntesdepsicologia.es/index.php/revista/article/view/53.

8. Rossi A, Panzeri A, Pietrabissa G, Manzoni GM, Castelnuovo G, Mannarini S. The Anxiety-Buffer Hypothesis in the Time of COVID19: When Self-Esteem Protects from the Impact of Loneliness and Fear on Anxiety and Depression. Front Psychol (2020) 11:2177. doi:10.3389/ fpsyg.2020.02177

9. Diener E, Biswas-Diener R. Happiness: Unlocking the Mysteries of Psychological Wealth. Malden, MA: Blackwell Publishing (2008).

10. Machado L, Tavares H, Petribú K, Zilberman M, Torres RF, Cantilino A. Happiness and Health in Psychiatry: What Are Their Implications. Arch Clin Psychiatry (São Paulo) (2015) 42(4):100-10. doi:10.1590/010160830000000058

11. Colucci E, Nadeau S, Higgins J, Kehayia E, Poldma T, Saj A, et al. COVID-19 Lockdowns' Effects on the Quality of Life, Perceived Health and Well-Being of Healthy Elderly Individuals: A Longitudinal Comparison of Prelockdown and Lockdown States of Well-Being. Arch Gerontol Geriatr (2022) 99:104606. doi:10.1016/j.archger.2021.104606

12. World Health Organization. Mental Health: Strengthening Our Response (2018). Available from: https://www.who.int/news-room/fact-sheets/detail/ mental-health-strengthening-our-response.

13. Hone LC, Jarden A, Schofield GM, Duncan S. Measuring Flourishing: The Impact of Operational Definitions on the Prevalence of High Levels of Wellbeing. Intnl J Wellbeing (2014) 4(1):62-90. doi:10.5502/ ijw.v4i1.4

14. Keyes CLM. The Mental Health Continuum: From Languishing to Flourishing in Life. J Health Soc Behav (2002) 43(2):207-22. doi:10.2307/3090197

\section{CONFLICT OF INTEREST}

The authors declare that the research was conducted in the absence of any commercial or financial relationships that could be construed as a potential conflict of interest.

15. Sürücü L, Ertan ŞS, Bağlarbaşı E, Maslakçı A. COVID-19 and Human Flourishing: The Moderating Role of Gender. Pers Individ Dif (2021) 183 111111. doi:10.1016/j.paid.2021.111111

16. Rosenberg M. Society and the Adolescent Self-Image. Princeton, NJ: Princeton University Press (1965).

17. Diener E, Wirtz D, Tov W, Kim-Prieto C, Choi D-w., Oishi S, et al. New Well-Being Measures: Short Scales to Assess Flourishing and Positive and Negative Feelings. Soc Indic Res (2010) 97:143-56. doi:10.1007/s11205009-9493-y

18. Checa I, Perales J, Espejo B. Spanish Validation of the Flourishing Scale in the General Population. Curr Psychol (2018) 37(4):949-56. doi:10.1007/s12144017-9581-0

19. Torres C, Otero P, Bustamante B, Blanco V, Díaz O, Vázquez FL. Mental Health Problems and Related Factors in Ecuadorian College Students. Int J Environ Res Public Health (2017) 14:530. doi:10.3390/ ijerph 14050530

20. Hendriksen PA, Garssen J, Bijlsma EY, Engels F, Bruce G, Verster JC. COVID19 Lockdown-Related Changes in Mood, Health and Academic Functioning. Ejihpe (2021) 11:1440-61. doi:10.3390/ejihpe11040103

21. Hidalgo-Fuentes S, Martínez-Álvarez I, Tijeras-Iborra A, Sospedra-Baeza MJ. Relación entre la inteligencia emocional y el apoyo social con el bienestar subjetivo: Un estudio transcultural España-Ecuador. Rev Psicol La Salud (2021) 10(1):41-53. 10.21134/pssa.v10i1.800.

22. Helliwell JF, Huang H, Wang S, Nortron M. "Happiness, Trust, and Deaths under COVID-19", in World Happiness Report. Editors J Helliwell, R Layard, JD Sachs, J De Neve, L Aknin, S Wang, et al. New York, NY: Sustainable Development Solutions Network (2021):15-56.

23. Country Economy. Ecuador-world Happiness Index (2021). Available from: https://countryeconomy.com/demography/world-happiness-index.

24. Bélanger RE, Patte KA, Leatherdale ST, Gansaonré RJ, Haddad S. An Impact Analysis of the Early Months of the COVID-19 Pandemic on Mental Health in a Prospective Cohort of Canadian Adolescents. J Adolesc Heal (2021) 69(6): 917-24. doi:10.1016/j.jadohealth.2021.07.039

25. Knoesen R, Naudé L. Experiences of Flourishing and Languishing during the First Year at university. J Ment Health (2018) 27(3):269-78. doi:10.1080/ 09638237.2017.1370635

26. Taylor MR, Agho KE, Stevens GJ, Raphael B. Factors Influencing Psychological Distress during a Disease Epidemic: Data from Australia's First Outbreak of Equine Influenza. BMC Public Health (2008) 8:347-13. doi:10.1186/14712458-8-347

27. Pieh C, Budimir S, Probst T. The Effect of Age, Gender, Income, Work, and Physical Activity on Mental Health during Coronavirus Disease (COVID-19) Lockdown in Austria. J Psychosomatic Res (2020) 136:110186. doi:10.1016/j. jpsychores.2020.110186

28. Horton R. Offline: COVID-19 Is Not a Pandemic. Lancet (2020) 396(10255): 874. doi:10.1016/S0140-6736(20)32000-6

29. Wang C, Pan R, Wan X, Tan Y, Xu L, McIntyre RS, et al. A Longitudinal Study on the Mental Health of General Population during the COVID-19 Epidemic in China. Brain Behav Immun (2020) 87(April):40-8. doi:10.1016/j.bbi.2020. 04.028

30. Twenge JM, Campbell WK. Associations between Screen Time and Lower Psychological Well-Being Among Children and Adolescents: Evidence from a Population-Based Study. Prev Med Rep (2018) 12(September):271-83. doi:10. 1016/j.pmedr.2018.10.003

31. Escobar-Viera CG, Shensa A, Bowman ND, Sidani JE, Knight J, James AE, et al. Passive and Active Social media Use and Depressive Symptoms Among 
United States Adults. Cyberpsychology, Behav Soc Networking (2018) 21(7): 437-43. doi:10.1089/cyber.2017.0668

32. Takeda F, Noguchi H, Monma T, Tamiya N. How Possibly Do Leisure and Social Activities Impact Mental Health of Middle-Aged Adults in Japan?: An Evidence from a National Longitudinal Survey. PLoS One (2015) 10(10): e0139777. doi:10.1371/journal.pone.0139777

33. Fancourt D, Opher S, de Oliveira C. Fixed-effects Analyses of Time-Varying Associations between Hobbies and Depression in a Longitudinal Cohort Study: Support for Social Prescribing. Psychother Psychosom (2020) 89(2):111-3. doi:10.1159/000503571

34. Fullana MA, Hidalgo-Mazzei D, Vieta E, Radua J. Coping Behaviors Associated with Decreased Anxiety and Depressive Symptoms during the COVID-19 Pandemic and Lockdown. J Affective Disord (2020) 275:80-1. doi:10.1016/j.jad.2020.06.027

35. Venkatesh A, Edirappuli S. Social Distancing in Covid-19: What Are the Mental Health Implications. BMJ (2020) 369(April):m1379. doi:10.1136/bmj.m1379
36. Greenberg J, Pyszczynski T, Solomon S. The Causes and Consequences of a Need for Self-Esteem: A Terror Management Theory. Public Self Private Self Springer Ser Soc Psychol (1984) 13 189-212.

37. Pyszczynski T, Greenberg J, Solomon S, Arndt J, Schimel J. Why Do People Need Self-Esteem? A Theoretical and Empirical Review. Psychol Bull (2004) 130(3):435-68. doi:10.1037/0033-2909.130.3.435

Copyright (C) 2022 Paz, Hermosa-Bosano, Hidalgo-Andrade, García-Manglano, Sábada Chalezquer, López-Madrigal and Serrano. This is an open-access article distributed under the terms of the Creative Commons Attribution License (CC BY). The use, distribution or reproduction in other forums is permitted, provided the original author(s) and the copyright owner(s) are credited and that the original publication in this journal is cited, in accordance with accepted academic practice. No use, distribution or reproduction is permitted which does not comply with these terms. 